INSTITUTSVORSTELLUNG

\title{
Das Zentrum für systemische Neuro- wissenschaften (ZSN) in Hannover
}

Wolfgang Löscher

Der Hochschulstandort Hannover bietet ein breites Spektrum an neurowissenschaftlichen Arbeitsfeldern. Die system- und verhaltensorientierten Neurowissenschaften profitieren dabei von einer Reihe von Standortvorteilen. Dazu gehören Abteilungen und Arbeitsgruppen in der Tierärztlichen Hochschule Hannover (TiHo), der Medizinischen Hochschule Hannover (MHH), der Universität Hannover, der Hochschule für Musik und Theater und dem Max-Planck-Institut für experimentelle Endokrinologie, die bereits seit 1994 im Rahmen des informellen "Arbeitskreises für Experimentelle und Klinische Neurowissenschaften Hannover", dem rund zwanzig Arbeitsgruppen angehörten, durch gemeinsame Vortragsveranstaltungen und wissenschaftliche Kooperation unterschiedlichen Grades hervorgetreten sind. Seit September 2002 hat Hannover nun ein Zentrum für systemische Neurowissenschaften (ZSN). Die Gründung dieses virtuellen Zentrums unter einheitlicher Kooperation wurde durch eine vom niedersächsischen Wissenschaftsministerium eingesetzte Strukturkommission angeregt, die nach rund dreijähriger Evaluation der biologischen und biomedizinischen Forschung und Lehre in Niedersachsen empfahl, in Hannover einen hochschulübergreifenden Schwerpunkt systemische Neurowissenschaften zu bilden, um das in Hannover bestehende Potential in diesem Bereich zu bündeln. Mit der Gründung des ZSN durch MHH, TiHo, Universität Hannover und Hochschule für Musik und Theater Hannover wurde dieser Empfehlung gefolgt.

Das ZSN besteht aus einem Kernbereich und einem Ringbereich. Zum Kernbereich gehören zur Zeit zwölf Arbeitsgruppen, die wesentlich an der Gründung des ZSN und der Etablierung seiner Aktivitäten sowie des vom ZSN seit Wintersemester 2003/04 durchgeführten PhD-Studiengangs systemische Neurowissenschaften beteiligt waren. Zum Ringbereich gehören zur Zeit zehn ausgewiesene neurowissenschaftliche Arbeitsgruppen und Nachwuchsgruppen an universitären Institutionen, dem Max-Planck-Insti- tut für experimentelle Endokrinologie und dem International Neuroscience Institute (INI) in Hannover. Die Themenschwerpunkte der Arbeitsgruppen von Kern- und Ringbereich des ZSN decken ein weites Spektrum der modernen Neurowissenschaften, vor allem in der system- und verhaltensbezogenen Neurobiologie, ab und reichen von der Systemphysiologie des Nervensystems, Mechanismen von Neurodegeneration und neuronaler Regeneration, Neurotransplantation, Neurogenetik, Verhaltensphysiologie und Neuropharmakologie über die klinische Neurophysiologie hin zur Musikphysiologie und der neurologischen und psychiatrischen Kognitionsforschung. Die Forschungsprofile der Arbeitsgruppen sowie weitere Details zum ZSN sind auf der Homepage des ZSN (www.zsn-hannover.de) dargestellt.

Die Koordination der Aktivitäten des ZSN erfolgt durch einen Vorstand, der sich aus Professoren der MHH (Claudia Grothe, Reinhard Dengler), TiHo (Wolfgang Löscher, Elke Zimmermann), Universität Hannover (Hans-Albert Kolb) und Musikhochschule Hannover (Eckart Altenmüller) zusammensetzt. Ein wissenschaftlicher Beirat berät das ZSN in allen wissenschaftlichen und strukturellen Fragen. Dem Beirat gehören die folgenden Wissenschaftler an: Prof. Dr. Michael Frotscher (Freiburg), Prof. Dr. Hans-Jochen Heinze (Magdeburg), Prof. Dr. Gerhard Neuweiler (München), Prof. Dr. Melitta Schachner (Hamburg) und Prof. Dr. Henning Scheich (Magdeburg).

Das ZSN verfolgt vor allem vier Ziele: (1) Bündelung der Forschung auf dem Gebiet der systemischen (systembezogenen) Neurowissenschaften mit der Absicht, eine höhere Wettbewerbsfähigkeit zu erreichen. (2) Stärkung der Forschung im Überlappungsbereich zwischen neurowissenschaftlicher Grundlagenforschung und klinischer Forschung mit der Absicht, durch die Verbindung von angewandter klinischer Forschung, neurobiologischer Grundlagenforschung und Neurotechnologie neue Impulse und Strategien für die Wiederherstellung von Funktionen im
Nervensystem zu entwickeln. (3) Etablierung von koordinierten neurowissenschaftlichen Forschungsschwerpunkten unter Berücksichtigung der besonderen Stärken der in Hannover tätigen Neurowissenschaftler. (4)

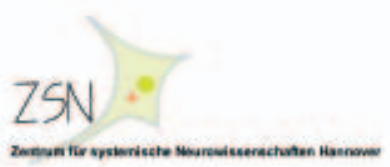

Strukturierte Ausbildung des wissenschaftlichen Nachwuchses durch Organisation und Durchführung eines eigenständigen Promotions-(PhD-)Studiums für Studierende der Biologie, Tiermedizin, Humanmedizin, Biochemie sowie verwandter naturwissenschaftlicher Studiengänge.

Das ZSN hat sich drei Forschungsschwerpunkte gegeben, die der thematischen Fokussierung und Profilbildung dienen sollen: (1) Neuroakustik und Emotion: Von den physiologischen Grundlagen zu komplexen Hirnleistungen und ihren Störungen (Koordination: Prof. Elke Zimmermann). (2) Charakterisierung genetisch induzierter Hirnveränderungen bei Tiermodellen: Anatomie, Physiologie und Verhalten (Koordination: Prof. W. Löscher); und (3) Wiederherstellung neuronaler Funktionen bei Bewegungsstörungen (Koordination Prof. Dr. E. Altenmüller). Diese drei Forschungsschwerpunkte des ZSN werden durch erhebliche Drittmittel von DFG, BMBF und EU gefördert. Außerdem wurden seit Gründung des ZSN zwei neue Forschergruppen der DFG zu den Schwerpunktthemen des ZSN in Hannover eingerichtet.

Das ZSN stellte kurz nach seiner Gründung einen Antrag an das niedersächsische Wissenschaftsministerium auf Förderung der Einrichtung eines neuen internationalen Promotionsstudienganges "Systemische Neurowissenschaften" im Rahmen der Innovationsoffensive des Landes zur Förderung von Promotionsprogrammen. Nach positiver Begutachtung durch die Wissenschaftliche Kommission Niedersachsen wurde der Antrag im Juni 2003 durch das MWK genehmigt und Mittel für Stipendien, ein Koordinationssekretariat und Reise- sowie Sachmittel zur Verfügung gestellt. Das dreijährige PhD-Programm (Studienordnung s. Homepage des ZSN), das mit dem Wintersemester 2003/04 aufgenommen wurde, soll einen wesentlichen Beitrag zur Ausbildung des wissenschaftlichen Nachwuchses auf dem Gebiet der systembezogenen Neurowissenschaften leisten und den Studierenden die Fähigkeit vermitteln, die sie für eine erfolgreiche Karriere in 
der Forschung benötigen. Die Studienplätze werden international ausgeschrieben, und aus den eingegangenen Bewerbungen wählt das ZSN nach Leistungskriterien jährlich 20 Stipendiaten und Kollegiaten für den Promotionsstudiengang aus. Der englischsprachige $\mathrm{PhD}$-Studiengang wird von einer vom Vorstand des ZSN eingesetzten Studienkommission und den Mitarbeiterinnen des Koordinationssekretariats organisiert.

Zusammengenommen ist durch die Gründung des ZSN und seiner vielfältigen Aktivitäten das Gewicht der Neurowissenschaf- ten in Hannover deutlich gestiegen. Wichtig für die weitere Entwicklung des ZSN ist die Wiederbesetzung zur Zeit vakanter neurowissenschaftlicher Lehrstühle (Neurophysiologie und Neuropathologie der $\mathrm{MHH}$ ) trotz der Sparbeschlüsse des Landes Niedersachsen. Aufgrund der erheblichen Bedeutung der systemorientierten Neurowissenschaften für die gegenwärtige und zukünftige biomedizinische und klinische Forschung hoffen die Hannoveraner Neurowissenschaftler auf eine weitere Stärkung dieses Wissenschaftsbereichs am Hochschulstandort Hannover.

\section{Die ethischen Herausforderungen der Neurowissenschaften}

Ethische und wissenschaftstheoretische Aspekte der Neurowissenschaften - Zur Einrichtung des Graduiertenkollegs „Bioethik“ am Interfakultären Zentrum für Ethik in den Wissenschaften (IZEW) der Universität Tübingen

\section{Eve-Marie Engels}

Am Interfakultären Zentrum für Ethik in den Wissenschaften (IZEW) der Universität Tübingen hat im Januar 2004 ein von der DFG gefördertes Graduiertenkolleg „Bioethik“ seine Arbeit aufgenommen. Das Thema „Ethische und wissenschaftstheoretische Aspekte der Neurowissenschaften" bildet einen der Forschungsschwerpunkte dieses Kollegs. Die Neurowissenschaften und ihre Technologien haben in den vergangenen Jahrzehnten beeindruckende Erkenntnisfortschritte in theoretischer und praktischer Hinsicht erzielt und uns faszinierende Einblikke und therapeutische Eingriffsmöglichkeiten in Gehirn und Nervensystem eröffnet. Große Hoffnungen werden in die Entwicklung weiterer Behandlungsmöglichkeiten von Patienten ${ }^{1}$ mit neuronalen Erkrankungen gesetzt. Gleichzeitig stellen uns diese Wissenschaften vor neuartige Herausforderungen. Sie beinhalten ethische, wissenschaftstheoretische, anthropologische und andere philosophische Implikationen mit weitreichenden Konsequenzen für Individuum und Gesellschaft. Dies liegt in der Besonderheit ihres Gegenstandes begründet. Als dem zentralen Steuerungsorgan für alle lebenswichtigen Leistungen und Funktionen kommt unserem Gehirn eine ausgezeichnete Bedeutung für die Gesamtheit unserer
Existenz und Lebensvollzüge zu. Unser Gehirn ist die Voraussetzung für die Möglichkeit von Selbstbewusstsein, für die Erfahrung personaler Identität, Willensfreiheit und Selbstbestimmung (Autonomie). Nicht nur Eingriffe in das Gehirn, sondern auch die Entwicklung von Theorien über dessen Leistungsvermögen und über die Beziehung zwischen Gehirn und Geist erfordern daher ein hohes Maß an Fingerspitzengefühl, Umsicht und ethischem Urteilsvermögen. Im Unterschied zu anderen Bereichsethiken der Bioethik, die seit längerem etabliert sind, gibt es eine Ethik der Neurowissenschaften (,Neuroethik") bisher jedoch noch nicht. Auch in der Öffentlichkeit werden ethisch relevante Aspekte dieser Entwicklungen bisher zu wenig wahrgenommen. Das Graduiertenkolleg „Bioethik“ soll bei der Konstituierung einer Neuroethik wichtige Weichenstellungen leisten und dabei auch die Aufgabe eines Sensors möglicher zukünftiger Chancen und Risiken der Neurowissenschaften wahrnehmen. Damit soll verhindert werden, dass die Ethik hinter neurowissenschaftlichen Entwicklungen ,herhinkt“", statt diese antizipierend und begleitend mitzubestimmen.

\section{Korrespondenzadresse}

\section{Prof. Dr. Wolfgang Löscher}

Sprecher des Zentrums für systemische

Neurowissenschaften Hannover

Institut für Pharmakologie, Toxikologie und Pharmazie

Tierärztliche Hochschule Hannover

Bünteweg 17

D-30559 Hannover

Tel.: ++49(0) 9538720

Fax: ++49 (0) 9538581

e-mail: wolfgang.loescher@tiho-hannover.de
Zur Notwendigkeit einer Institutionalisierung bioethischer Kompetenz

Etwa seit der zweiten Hälfte des 20. Jahrhunderts gibt es einen ständig wachsenden öffentlichen Bedarf an einer Ethik der Wissenschaften. Dabei kristallisieren sich die Lebenswissenschaften, d.h. Biologie, Medizin und ihre Technologien, zunehmend als Anlässe und zentrale Gegenstände ethischer und rechtlicher Reflexion heraus. Mit den spektakulären Entwicklungen in einzelnen ihrer Bereiche und Anwendungsmöglichkeiten werden Handlungsspielräume eröffnet, die Ethik, Philosophie und Recht mit neuen Fragen konfrontieren. Diese Handlungsoptionen stellen uns fortwährend vor die Notwendigkeit, ethische und rechtliche Entscheidungen darüber zu treffen, wie wir mit ihnen umgehen wollen und sollen. Wir haben gar keine andere Wahl, als uns hier zu positionieren und dabei sorgfältige Abwägungen unter Berücksichtigung aller relevanten Aspekte vorzunehmen. Hierfür ist die Herausbildung eines möglichst fundierten bioethischen Urteilsvermögens erforderlich.

Zwischen dem allgemein anerkannten Bedarf an bioethischer Kompetenz und den verfügbaren Ausbildungsmöglichkeiten im Bereich der Bioethik besteht jedoch nach wie vor eine erhebliche Kluft. Hauptgrund hierfür ist unser Ausbildungssystem. Eine interdisziplinäre Kompetenz, wie sie für eine fundierte bioethische Urteilsbildung erforderlich ist, lässt sich über die etablierten Studiengänge nur schwer erlangen.

Diese Kompetenz setzt vielmehr die Struktur einer interdisziplinären Kooperation voraus, in der Vertreter natur- und geisteswissenschaftlicher Disziplinen und der Medizin an gemeinsamen Fragestellungen arbeiten. Ein interdisziplinäres Graduiertenkolleg „Bioethik“ bietet daher durch sein Forschungs- und Ausbildungsprogramm die 